\title{
Validation of Heart Failure Events in the Antihypertensive and Lipid Lowering Treatment to Prevent Heart Attack Trial (ALLHAT) Participants Assigned to Doxazosin and Chlorthalidone Linda B Piller*1, Barry R Davis1, Jeffrey A Cutler ${ }^{2}$, William C Cushman², Jackson T Wright Jr4, Jeff D Williamson ${ }^{5}$, Frans HH Leenen ${ }^{6}$, Paula T Einhorn ${ }^{2}$, Otelio S Randall7, John S Golden ${ }^{8}$ and L Julian Haywood ${ }^{9}$ for the ALLHAT Collaborative Research Group ${ }^{10}$
}

\begin{abstract}
Address: ${ }^{1}$ The University of Texas School of Public Health, Houston, TX, USA, ${ }^{2}$ National Heart, Lung, and Blood Institute, Bethesda, MD, USA, ${ }^{3}$ Memphis Veterans Affairs Medical Center, Memphis, TN, USA, ${ }^{4}$ Case Western Reserve University School of Medicine, Cleveland, OH, USA, ${ }^{5}$ Wake Forest University, Winston-Salem, NC, USA, ${ }^{6}$ University of Ottawa Heart Institute, Ottawa, ON, Canada, ${ }^{7}$ Howard University Medical School, Washington, DC, USA, ${ }^{8}$ Kaiser Permanente, Mid-Atlantic States, Washington, DC, USA, ${ }^{9}$ Keck School of Medicine, University of Southern California, Los Angeles, CA, USA and ${ }^{10}$ For a complete list of members of the ALLHAT Collaborative Research Group see JAMA 2000; 283:19731975
\end{abstract}

E-mail: Linda B Piller* - lpiller@sph.uth.tmc.edu; Barry R Davis - bdavis@sph.uth.tmc.edu; Jeffrey A Cutler - CUTLERJ@NHLBI.NIH.GOV; William C Cushman - WILLIAM.CUSHMAN@MED.VA.GOV; Jackson T Wright - JXW20@PO.CWRU.EDU;

Jeff D Williamson - JWILLIAM@WFUBMC.EDU; Frans HH Leenen - fleenen@ottawaheart.ca; Paula T Einhorn - einhornp@nhlbi.nih.gov; Otelio S Randall - osrandall@ howard.edu; John S Golden - John.Golden@kp.org; L Julian Haywood - JHAYWOOD@HSC.USC.EDU; the ALLHAT Collaborative Research Group - bdavis@sph.uth.tmc.edu

${ }^{*}$ Corresponding author

Published: 14 November 2002

Current Controlled Trials in Cardiovascular Medicine 2002, 3:10

This article is available from: http://cvm.controlled-trials.com/content/3/1/10

(c) 2002 Piller et al; licensee BioMed Central Ltd. This article is published in Open Access: verbatim copying and redistribution of this article are permitted in all media for any purpose, provided this notice is preserved along with the article's original URL.

Keywords: heart failure, alpha-blocker, diuretic, clinical trial
Received: 23 October 2002

Accepted: 14 November 2002

\begin{abstract}
Keywords: heart failure, alpha-blocker, diuretic, clinical tial
\end{abstract}
\begin{abstract}
Background: The Antihypertensive and Lipid Lowering Treatment to Prevent Heart Attack Trial (ALLHAT) is a randomized, double-blind, active-controlled trial designed to compare the rate of coronary heart disease events in high-risk hypertensive participants initially randomized to a diuretic (chlorthalidone) versus each of three alternative antihypertensive drugs: alpha-adrenergic blocker (doxazosin), ACE-inhibitor (lisinopril), and calcium-channel blocker (amlodipine). Combined cardiovascular disease risk was significantly increased in the doxazosin arm compared to the chlorthalidone arm (RR I.25; 95\% Cl, I.I7-I.33; $P<.001)$, with a doubling of heart failure (fatal, hospitalized, or non-hospitalized but treated) (RR 2.04; 95\% Cl, I.79-2.32; $P<.00 \mathrm{I}$ ). Questions about heart failure diagnostic criteria led to steps to validate these events further.
\end{abstract}

Methods and Results: Baseline characteristics (age, race, sex, blood pressure) did not differ significantly between treatment groups $(P<.05)$ for participants with heart failure events. Postevent pharmacologic management was similar in both groups and generally conformed to accepted heart failure therapy. Central review of a small sample of cases showed high adherence to ALLHAT heart failure criteria. Of 105 participants with quantitative ejection fraction measurements provided, (67\% by echocardiogram, $31 \%$ by catheterization), $29 / 46(63 \%)$ from the chlorthalidone group and $41 / 59(70 \%)$ from the doxazosin group were at or below $40 \%$. Two-year heart failure case-fatalities (22\% and 19\% in the doxazosin and chlorthalidone groups, respectively) were as expected and did not differ significantly (RR 0.96; $95 \% \mathrm{Cl}, 0.67-1.38 ; P=0.83)$. 
Conclusion: Results of the validation process supported findings of increased heart failure in the ALLHAT doxazosin treatment arm compared to the chlorthalidone treatment arm.

The Antihypertensive and Lipid Lowering Treatment to Prevent Heart Attack Trial (ALLHAT) is a randomized, two-component clinical trial sponsored by the National Heart, Lung, and Blood Institute (NHLBI). A doubleblind, active-controlled hypertension component is designed to compare the rate of fatal coronary heart disease (CHD) or nonfatal myocardial infarction (MI) (the primary endpoint) in high-risk hypertensive participants, aged 55 years or older, between those randomized to treatment initiated with a diuretic (chlorthalidone) and treatment initiated with each of three alternative antihypertensive drugs: a calcium-channel blocker (amlodipine), an angiotensin-converting enzyme (ACE)inhibitor (lisinopril), or an alpha-adrenergic blocker (doxazosin). An open-label lipid-lowering component is designed to determine if lowering LDL cholesterol with pravastatin compared to "usual care" reduces all-cause mortality in a subset of moderately hypercholesterolemic patients. Randomization to the hypertension component began in February, 1994, and continued through January, 1998, with 42,418 participants recruited at 623 clinical centers in the United States, Canada, Puerto Rico and the US Virgin Islands. Randomization of 10,355 participants into the lipid trial ended May 31, 1998. Follow-up on all participants continued through March, 2002 [1].

Following independent reviews by the Data and Safety Monitoring Board (DSMB) on January 6, 2000, and by an Ad Hoc Committee on January 21, 2000, the Director of the National Heart, Lung, and Blood Institute accepted a recommendation to discontinue the doxazosin treatment arm of the antihypertensive trial. This recommendation was based on the low probability of doxazosin showing benefit over chlorthalidone for the primary endpoint, as well as the significantly increased occurrence of the secondary endpoint, combined cardiovascular disease (CVD) (encompassing CHD deaths, nonfatal MI, stroke, coronary revascularization procedures [coronary artery bypass graft or CABG, percutaneous transluminal coronary angioplasty or PTCA/stent], angina [hospitalized or treated as an outpatient], heart failure [HF/treated in the hospital or as an outpatient], and peripheral arterial disease [in-hospital or outpatient revascularization]) in the doxazosin arm (RR 1.25; 95\% CI, 1.17-1.33; $P<.001$ ), with a doubling of risk of HF (fatal, hospitalized and treated but nonhospitalized) (RR 2.04; 95\% CI, 1.79-2.32; $P$ $<.001)$. When only fatal and hospitalized HF were analyzed, the large difference remained (RR 1.83; 95\% CI, $1.58-2.13 ; P<.001)$. The findings and operational aspects of stopping the doxazosin arm of the study have been previously described [2-4].

The observed increase in HF in the doxazosin group as compared to the chlorthalidone group led to several additional analyses aimed toward validation of the diagnoses, with a focus on hospitalized and fatal HF. The purposes of these analyses were: 1) to evaluate the reality of HF cases, i.e. whether diagnosis, management, and clinical course were what might be expected, and 2) to compare these features between the two treatment groups.

\section{Methods Study Design}

The rationale and design of ALLHAT are described in detail elsewhere [1]. Briefly, those eligible for randomization had systolic blood pressure (SBP) of at least $140 \mathrm{~mm}$ $\mathrm{Hg}$ and/or diastolic blood pressure of at least $90 \mathrm{~mm} \mathrm{Hg}$, or took medication for hypertension, and had at least one other risk factor for CHD events. Risk factors included previous MI or stroke, left ventricular hypertrophy by electrocardiogram or echocardiogram, history of type 2 diabetes, current cigarette smoking, and low high-density lipoprotein (HDL) level.

The primary endpoint of the blood pressure (BP) trial is the composite of nonfatal MI and fatal CHD. The four protocol-defined secondary clinical outcomes are allcause mortality, combined CHD (including CHD death, nonfatal MI, coronary revascularization procedures and hospitalized angina), stroke, and combined CVD (including CHD death, nonfatal MI, stroke, coronary revascularization procedures, angina treated in the hospital or as an outpatient, lower extremity peripheral arterial disease treated in the hospital or with outpatient revascularization, and HF, fatal or treated in the hospital or as an outpatient).

The validation of HF diagnosis entailed answering several questions:

\section{Did HF cases meet ALLHAT diagnostic criteria?}

2. Were baseline characteristics and medical management for $\mathrm{HF}$ cases as expected and similar across the drug groups?

3. Were prevalence and severity of systolic dysfunction as expected and similar across drug groups? 
4. Were case-fatality rates as expected and similar across drug groups?

Unless otherwise specified, all data for these analyses were collected as of December, 1999.

\section{Heart Failure Diagnosis}

At each follow-up clinic visit the occurrence of study clinical events was assessed, and, if identified by the clinical investigator, reported on an endpoint form. For each event involving hospitalization, a hospital discharge summary or expiration summary was to be submitted, and for each death a death certificate was required. Endpoint forms and supporting documentation were reviewed at the ALLHAT Clinical Trials Center (CTC) for accuracy and appropriateness. When a discrepancy or ambiguity was found, the CTC sent a written query to the Principal Investigator, who retained the final word concerning the diagnosis or cause of death. A random 10\% sample of strokes, nonfatal MIs and CHD deaths was selected for blinded quality control evaluation by the ALLHAT Endpoints Subcommittee; for these cases additional documentation was requested.

No such routine Endpoints Subcommittee quality control was initially planned for reported HF. However, the Subcommittee was subsequently called upon to evaluate a random sample of reported fatal and hospitalized nonfatal HF events. As this occurred prior to the termination of the doxazosin arm, neither the chair nor the members of the Subcommittee was informed of the major reason for this review, namely, the trend toward a higher HF event rate in the doxazosin group compared to the chlorthalidone group. The Subcommittee was told that the review was undertaken at the request of the DSMB to address the reliability and validity of reported HF events. This evaluation consisted of fifty events, evenly distributed across the four treatment groups, reported as fatal or hospitalized nonfatal HF and with protocol-required documentation (discharge summary for hospitalized events, death certificates for deaths). Additional material was not requested, since this would have posed an undue burden on the clinical site staff and would have risked raising questions about emerging differences among treatment groups.

The ALLHAT definition of HF, used previously in the Systolic Hypertension in the Elderly Program (SHEP) [5], includes "patients with clear-cut signs or symptoms of left or right ventricular dysfunction that cannot be attributed to other causes..." The diagnosis of HF must include at least one of four stated symptoms [paroxysmal nocturnal dyspnea, dyspnea at rest, New York Heart Classification functional class III (for definition see Additional Information, Item 1), or orthopnea], and one of seven stated signs (rales, 2+ or greater ankle edema, tachycardia of 120 beats/minute or more after five minutes at rest, cardiomegaly by chest $\mathrm{x}$-ray, chest $\mathrm{x}$-ray characteristic of $\mathrm{HF}, \mathrm{S}_{3}$ gallop, or jugular venous distention). Since lower extremity edema or exertional dyspnea may be due to non-cardiac causes, the presence of either of these alone, without other indications of heart failure, is not sufficient for a diagnosis of HF. Study guidelines caution against a hasty HF diagnosis in patients with severe pulmonary disease, including chronic obstructive pulmonary disease (COPD), pneumonia, or other severe, documented lung disease.

\section{Baseline Characteristics and Medical Management}

Baseline characteristics of chlorthalidone and doxazosin participants were compared. These analyses were stratified by HF outcome: 1) fatal and hospitalized HF; 2) treated, non-hospitalized HF; and, 3) no HF.

Post-HF event medical management may provide additional evidence of the physicians' confidence in the HF diagnosis. The post-event use of open-label diuretics, ACEinhibitors, and beta-blockers, i.e., accepted treatments for HF [6-9], was compared between doxazosin and chlorthalidone groups, as was the proportion in each group of those who remained on assigned blinded medication after the event. (For study guidelines regarding the use and reporting of open-label medicines of the same class as the blinded drugs, see Additional Information, Items 2 and 3.)

\section{Ejection Fraction Review}

A CTC physician plus non-medical staff reviewed in a blinded fashion 361 hospitalized HF events (representing 278 participants) for ejection fraction data: looking for whether an ejection fraction was measured, the method utilized, and the measurement. Results, tabulated by randomization groups, reflected data that had been collected up to July, 1999, the time of the review.

\section{Case-Fatality Rates and Causes of Death}

As a measure of the diagnostic validity and severity of HF and comparability between drug groups, time-fromevent-to-death analyses of participants with hospitalized or treated HF were compared between the two groups. Causes of death of such participants were also compared in the doxazosin and chlorthalidone groups.

\section{Statistical Analyses}

Data were analyzed according to participants' randomization assignments and HF outcome status, regardless of subsequent medication adherence. The Kaplan-Meier method was utilized in calculating cumulative event rates. Descriptive statistics by treatment groups were presented for baseline characteristics, HF ascertainment, ejection fractions and use of HF medications. Comparability of 
Table I: Baseline Characteristics of Participants With and Without Heart Failure

\begin{tabular}{|c|c|c|c|c|c|c|}
\hline & \multicolumn{2}{|c|}{ Hospitalized and Fatal HF } & \multicolumn{2}{|c|}{ Treated, Nonhospitalized HF } & \multicolumn{2}{|c|}{ Without HF* } \\
\hline & C & D & C & D & C & D \\
\hline No. of subjects & 327 & 346 & 93 & 145 & 14493 & 8347 \\
\hline Age, years, mean (SD) & $70.7(8.3)$ & $71.0(8.6)$ & $70.3(8.2)$ & $69.9(7.2)$ & $66.8(7.6)$ & $66.6(7.6)$ \\
\hline \multicolumn{7}{|l|}{ Race } \\
\hline White, non-Hispanic, \% & 61.2 & 55.8 & 63.4 & 64.8 & 47.3 & 46.3 \\
\hline Black, non-Hispanic, \% & 30.6 & 35.3 & 32.3 & 30.3 & 32.0 & 32.9 \\
\hline Hispanic, \% & 4.6 & 5.5 & 2.2 & 2.8 & 15.6 & 16.1 \\
\hline Other, \% & 3.7 & 3.1 & 2.2 & 2.1 & 5.1 & 4.8 \\
\hline Women, \% & 42.8 & 39.6 & 33.3 & 44.1 & 47.0 & 46.5 \\
\hline Education, years, mean (SD) & $10.5(3.9)$ & $10.7(3.4)$ & II.3 (3.6) & $12.1(3.2)$ & II.0 (4.0) & II.0 (4.0) \\
\hline Cigarette smoking, current, \% & 19.0 & 19.4 & 11.8 & 17.9 & 22.1 & 21.9 \\
\hline \multicolumn{7}{|l|}{ Antihypertensive treatment } \\
\hline Treated > 2 months, $\%$ & 86.9 & 86.8 & 95.7 & 95.2 & 86.9 & 86.7 \\
\hline Treated $<2$ months, $\%$ & 3.3 & 3.3 & 1.1 & 0.0 & 3.3 & 3.3 \\
\hline Untreated, \% & 9.8 & 10.0 & 3.2 & 4.8 & 9.9 & 10.0 \\
\hline Blood pressure, $\mathrm{mm} \mathrm{Hg}$, mean (SD) & $\begin{array}{l}|47.8 / 8| . \mid \\
(17.6 / 10.9)\end{array}$ & $\begin{array}{l}150.1 / 81.8 \\
(16.5 / \mid 1.0)\end{array}$ & $\begin{array}{l}147.1 / 82.2 \\
(16.0 / 10.5)\end{array}$ & $\begin{array}{l}145.9 / 82.0 \\
(15.8 / 10.6)\end{array}$ & $\begin{array}{l}\mid 46.2 / 84.1 \\
(15.6 / 10.0)\end{array}$ & $\begin{array}{c}\mid 46.1 / 84.1 \\
(15.7 / 10)\end{array}$ \\
\hline Pulse pressure, mm Hg, mean (SD) & $66.7(16.5)$ & $68.3(15.3)$ & $64.9(13.0)$ & $63.9(15.3)$ & $62.1(14.1)$ & $62.1(14.2)$ \\
\hline \multicolumn{7}{|l|}{ Eligibility risk factorst } \\
\hline \multicolumn{7}{|l|}{ LVH } \\
\hline By electrocardiogram, \% & 20.5 & 22.3 & 18.3 & 21.4 & 16.1 & 16.1 \\
\hline By echocardiogram, \% & 6.3 & 4.7 & 4.4 & 1.4 & 4.6 & 4.6 \\
\hline Old MI or stroke, \% & 36.1 & 34.7 & 41.9 & 33.1 & 23.1 & 22.3 \\
\hline CABG/Angioplasty, \% & 22.6 & 18.5 & 25.8 & 26.2 & 12.9 & 12.6 \\
\hline ASCVD, $\%$ & 33.3 & 29.2 & 31.2 & 31.0 & 23.4 & $24.6 \ddagger$ \\
\hline Type 2 diabetes, $\%$ & 46.8 & 48.3 & 43.0 & 40.7 & 35.9 & 34.7 \\
\hline $\mathrm{HDL}<35 \mathrm{mg} / \mathrm{dl}, \%$ & 12.8 & 13.6 & 14.0 & 13.8 & 11.9 & 11.7 \\
\hline ST-T wave changes, $\%$ & 12.8 & 10.6 & 10.9 & 12.9 & 10.4 & 10.2 \\
\hline
\end{tabular}

$\mathrm{HF}=$ Heart Failure $\mathrm{C}=$ Chlorthalidone treatment group $\mathrm{D}=$ Doxazosin treatment group mm $\mathrm{Hg}=$ millimeters mercury LVH = Left ventricular hypertrophy $\mathrm{MI}=$ Myocardial infarction CABG = Coronary artery bypass graft ASCVD = Atherosclerotic cardiovascular disease HDL = High-density lipoprotein *Only participants with at least one follow-up visit are included. 355 chlorthalidone and 229 doxazosin participants are excluded due to lack of at least one follow-up visit. FFor trial eligibility, participants had to have at least I other risk factor in addition to hypertension. The indicated risk factors are not mutually exclusive or exhaustive and do not represent prevalence. $\ddagger P=.0484$

baseline characteristics of the treatment and HF outcome groups was ascertained by the $\chi^{2}$ test for categorical variables and standard normal $(\mathrm{z})$ test for continuous variables.

\section{Results}

Heart Failure Diagnostic Criteria

The blinded review by the Endpoints Subcommittee of 50 fatal or hospitalized HF events from the 4 drug groups determined $11(22 \%)$ to have incomplete data for a definitive review. Of the remaining $39,33(85 \%)$ were confirmed to have HF by one or both reviewers. For both the chlorthalidone and doxazosin groups, the diagnosis of HF was confirmed in $90 \%(18 / 20)$.

\section{Baseline Characteristics}

Baseline characteristics for the doxazosin and chlorthalidone treatment groups, stratified for HF status, are described in Table 1.

Baseline characteristics of doxazosin and chlorthalidone participants with subsequent hospitalized or fatal HF were compared. Doxazosin participants with hospitalized or fatal HF had a higher baseline SBP than the chlorthalidone participants (150.1 vs. $147.8 \mathrm{~mm} \mathrm{Hg}$ at the randomization visit). More doxazosin participants had LVH by ECG $(22.3 \%$ vs. $20.5 \%$ of chlorthalidone participants); slightly more chlorthalidone than doxazosin participants had LVH by echocardiogram. Several eligibility risk factors, including previous MIs or strokes, coronary revascularization procedures, other atherosclerotic cardiovascular 
Table 2: Post-Event Pharmacologic Treatment of Participants With and Without Heart Failure

\begin{tabular}{|c|c|c|c|c|c|c|}
\hline & \multicolumn{2}{|c|}{ Hospitalized Heart Failure* } & \multicolumn{2}{|c|}{$\begin{array}{c}\text { Treated, Nonhospitalized } \\
\text { Heart Failure* }\end{array}$} & \multicolumn{2}{|c|}{ Without Heart Failure ${ }^{\dagger}$} \\
\hline & C & D & C & D & C & D \\
\hline No. of participants & 232 & 281 & 93 & 145 & 14493 & 8347 \\
\hline \multicolumn{7}{|l|}{ Post-event medication } \\
\hline Blinded medication, (\%) & $83(35.8)$ & $126(44.8)$ & $54(58.1)$ & $93(64.1)$ & I I 247 (77.6) & $6096(73.0)$ \\
\hline Open-label diuretic (\%) & $135(58.2)$ & $180(64.0)$ & $57(61.3)$ & $98(67.6)$ & $1279(8.8)$ & $1153(13.8)$ \\
\hline Open-label ACE-inhibitor (\%) & $90(38.8)$ & $114(40.5)$ & $31(33.3)$ & $44(30.3)$ & $1154(8.0)$ & $826(9.9)$ \\
\hline Open-label beta-blocker (\%) & $33(14.2)$ & $38(13.5)$ & $18(19.4)$ & $32(22.1)$ & $3149(21.7)$ & $2016(24.0)$ \\
\hline $\begin{array}{l}\text { Open-label diuretic, ACE-inhibitor } \\
\text { or beta-blocker (\%) }\end{array}$ & $169(72.8)$ & $210(74.8)$ & $72(77.4)$ & $119(82.1)$ & $4829(33.3)$ & $3347(40.1)$ \\
\hline
\end{tabular}

$C=$ Chlorthalidone treatment group $D=$ Doxazosin treatment group ACE $=$ Angiotensin converting enzyme *Represents data as of first clinic visit after event. $\dagger$ Represents data as of most recent clinic visit. All participants represented have at least one follow-up visit. 355 chlorthalidone and 229 doxazosin participants are excluded due to lack of at least one follow-up visit.

disease (ASCVD), and ST-T wave changes, were reported more often in chlorthalidone than in doxazosin participants, though the differences were not significant. Chlorthalidone and doxazosin participants with hospitalized or fatal HF had similar pre-trial antihypertensive treatment durations. None of these differences was significant at $P<.05$.

Participants with HF events displayed higher rates of most cardiovascular risk factors compared to those without HF events. Approximately 35-36\% of chlorthalidone and doxazosin participants with fatal or hospitalized HF had reported prior MI or stroke as baseline eligibility risk factors, compared to $22-23 \%$ of those without HF $(P<$ .001). Participants with hospitalized/fatal HF had significantly higher rates of pre-randomization coronary artery bypass grafts (CABGs) and coronary angioplasties (19$23 \%$ of those with HF vs. $13 \%$ of those without; $P<.001)$ and other atherosclerotic cardiovascular disease (ASCVD) (29-33\% of those with HF vs. $23-25 \%$ of those without; $P<.001)$. Diabetes as a baseline risk factor occurred more frequently in those with HF: $47-48 \%$ in those with HF, $35-36 \%$ in those without $(P<.001)$. Left ventricular hypertrophy (LVH) by ECG was a risk factor in $21-22 \%$ of those who later developed HF, compared with $16 \%$ of those in each group who did not develop HF $(P<.001)$.

Baseline pulse pressure (PP) showed some variation between participants who did and did not develop HF. For participants with subsequent hospitalization or death from HF, PP was 67 and $68 \mathrm{~mm} \mathrm{Hg}$ for the chlorthalidone and doxazosin groups, respectively. Treated, non-hospitalized participants showed a lower mean baseline PP of
65 (chlorthalidone group) and $64 \mathrm{~mm} \mathrm{Hg}$ (doxazosin group), and an even lower mean PP in those without HF (62 $\mathrm{mm} \mathrm{Hg}$ in each group).

\section{Medical Management}

Table 2 presents post-event pharmacologic treatment of participants with HF and antihypertensive treatment of participants without HF. Following hospitalization for HF, 36\% (83/232) of chlorthalidone participants and $45 \%(126 / 281)$ of doxazosin participants remained on their blinded medications. Percentages of participants on open-label diuretics and ACE-inhibitors were similar following the event: $58 \%(135 / 232)$ of chlorthalidone participants and $64 \%(180 / 281)$ of doxazosin participants were prescribed open-label diuretics; 39\% (90/232) of chlorthalidone participants and $41 \%(114 / 281)$ of doxazosin participants were prescribed open-label ACE-inhibitors. Beta-blockers were prescribed for $14 \%$ of each group following the event, which was actually somewhat less than for participants who did not develop HF. Three-quarters of hospitalized HF participants in each group (169/ 232 in chlorthalidone group, 210/281 in doxazosin group) received at least one of the three medications (diuretic, ACE-inhibitor, or beta-blocker) post-hospitalization.

Among participants treated but not hospitalized for HF, $58 \%(54 / 93)$ of the chlorthalidone group and 64\% (93/ 145 ) of the doxazosin group remained on their blinded medication. Over $60 \%$ of participants in each group were prescribed open-label diuretics and over 30\% received ACE-inhibitors. Open-label beta-blocker use post-event occurred in 19\% (18/93) of the chlorthalidone group and 
Table 3: Ejection Fraction Data for Participants Hospitalized with Heart Failure*

\begin{tabular}{|c|c|c|c|}
\hline & $\begin{array}{l}\text { Chlorthalidone Treatment } \\
\text { Group }\end{array}$ & $\begin{array}{l}\text { Doxazosin Treatment } \\
\text { Group }\end{array}$ & Total \\
\hline Participants with hospitalized heart failure & 245 & 296 & 541 \\
\hline Hospitalized heart failure participants reviewed & 134 & 144 & 278 \\
\hline $\begin{array}{l}\text { Total reviewed heart failure participants with quantitative } \\
\text { ejection fractionst }\end{array}$ & 46 & 59 & 105 \\
\hline By cardiac catheterization (\%) & II (23.9) & $21(35.6)$ & $32(30.5)$ \\
\hline By echocardiogram (\%) & $33(71.7)$ & $37(62.7)$ & $70(66.7)$ \\
\hline Method not given (\%) & $2(4.3)$ & $\mathrm{I}(\mathrm{I} .7)$ & $3(2.9)$ \\
\hline \multicolumn{4}{|l|}{ Ejection fraction $(\%)^{\ddagger}$} \\
\hline $1-30 \%$ & $15(32.6)$ & $27(45.8)$ & $42(40.0)$ \\
\hline $31-40 \%$ & $14(30.4)$ & $14(23.7)$ & $28(26.7)$ \\
\hline $4 I-50 \%$ & $9(19.6)$ & $7(11.9)$ & $16(15.2)$ \\
\hline $5 I-60 \%$ & $3(6.5)$ & $10(16.9)$ & $13(12.4)$ \\
\hline$>60 \%$ & $5(10.9)$ & $\mathrm{I}(\mathrm{I} .7)$ & $6(5.7)$ \\
\hline
\end{tabular}

*Evaluation of documentation for ejection fractions was completed in July, 1999, 6 months prior to the decision to discontinue the doxazosin arm of ALLHAT. Total numbers of events and participants with heart failure in Table 3 are based on data at the time of review. tEvent documentation

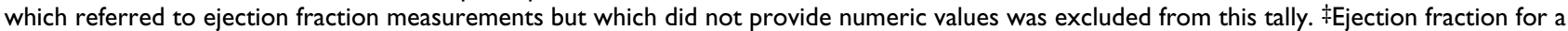
participant is based upon the earliest post-randomization heart failure hospitalization with quantitative ejection fraction information provided.

$22 \%$ (32/145) of the doxazosin group, approximately the same frequency as in participants without HF. In both treatment groups, a diuretic, ACE-inhibitor or beta-blocker was prescribed for over $75 \%$ of these participants (72/ 93 in chlorthalidone group, 119/145 in doxazosin group).

\section{Ejection Fraction Review}

Table 3 displays the ejection fraction data. About half $(178 / 361)$ of reviewed discharge summaries mentioned ejection fractions measured during hospitalization, twothirds (116/169) of which had quantitative measurements reported in the discharge summaries (data not given). Considering only the earliest ejection fraction information ascertained for each HF participant, 63\% (29/46) of the chlorthalidone and 70\% (41/59) of the doxazosin participants had ejection fractions at or below $40 \%$. Just under half $(27 / 59)$ of the ejection fractions reported in the doxazosin group were at or below $30 \%$, compared with one third (15/46) in the chlorthalidone group. Two-thirds of ejection fractions were obtained by echocardiograms, though catheterizations accounted for a larger percentage of results in the doxazosin group than in the chlorthalidone group (36\% vs. $24 \%$ ).

\section{Causes of Death and 2-Year Case-Fatality}

Causes of death of participants with previous HF hospitalization were distributed similarly in the two groups, with slight variations. HF accounted for $21.2 \%$ (11/52) of the deaths among chlorthalidone participants and $17.1 \%$ $(12 / 70)$ of the deaths among doxazosin participants. Over half of the deaths in each group (29/52 in the chlorthalidone group; 43/70 in the doxazosin group) were due to cardiovascular events. Fifteen percent (8/52) of deaths in the chlorthalidone group were attributed to cancer, compared to $9 \%(6 / 70)$ in the doxazosin group (Table 4$)$.

Case-fatality for participants with hospitalized HF events showed no significant differences (RR 0.96, 95\% CI, 0.67-1.38, $P=0.83$ ) between the two treatment groups (Figure 1). Among participants in the doxazosin treatment group who had been previously hospitalized for HF, $22.1 \%$ (70/317) subsequently died, compared to $18.6 \%$ $(52 / 280)$ of those in the chlorthalidone group (Table 4$)$. As previously reported, all-cause mortality did not significantly differ in the two treatment groups (RR 1.03, 95\% CI $0.90-1.15, P=0.56)$ [2].

\section{Discussion}

The finding of significantly increased HF events in the doxazosin group compared with the chlorthalidone group created a dilemma for ALLHAT. Since the trial was not designed to focus on HF, a component of a secondary endpoint, the validity of reported HF events became an issue. In this paper, we have described several analyses to address this concern.

As previously reported, lost-to-follow-up and event documentation were similar in the two treatment groups [2]. Steps employed to validate the HF outcome in these treatment groups confirmed the consistency of HF event reporting. Participants in the two drug groups had similar 
Table 4: Causes of Death of Participants with Prior Heart Failure Hospitalization

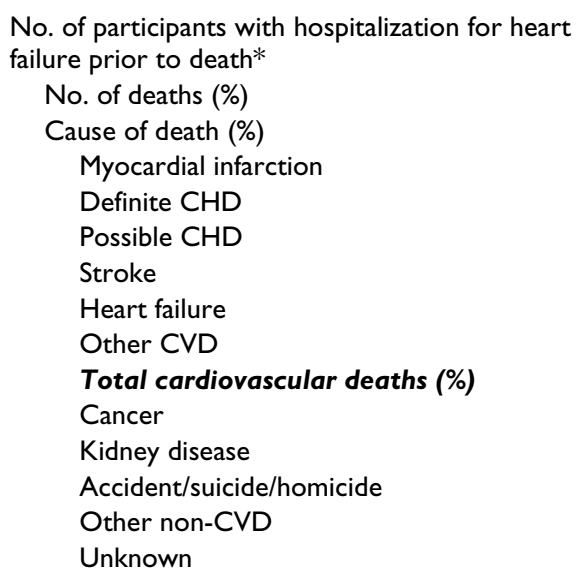

I (1.9)

I (I.9)

II (2I.2)

4 (7.7)

$29(55.8)$

8 (I5.4)

I (1.9)

$0(0)$

$8(15.4)$

$6(11.5)$
317

$70(22.1)$

$5(7.1)$

$9(12.9)$

$7(10.0)$

$3(4.3)$

$12(17.1)$

$7(10.0)$

$43(61.4)$

$6(8.6)$

I (I.4)

I (I.4)

II $(15.7)$

8 (II.4)

$\mathrm{CHD}=$ Coronary heart disease CVD = Cardiovascular disease $*$ Excludes participants for whom death is the first reported heart failure event

baseline characteristics when stratified by HF status. The differences in eligibility risk factors between those participants with HF and those without is not surprising: larger percentages of those with HF had a history of MI, stroke, CABG, angioplasty, other atherosclerotic cardiovascular disease (ASCVD), diabetes, low HDL levels and ECG abnormalities. Nonetheless, these eligibility factors were not substantially different between chlorthalidone and doxazosin participants with HF.

Post-diagnosis pharmacologic management of patients is one measure of the strength of physicians' confidence in the HF diagnoses. Open-label diuretics, ACE-inhibitors and beta-blockers, all recognized treatments for HF, were prescribed similarly for the chlorthalidone and doxazosin groups, suggesting similar assessment of these events in the two treatment groups.

ALLHAT criteria for HF were equivalently met in the two groups. While the sample $(\mathrm{n}=50)$ of hospitalized or fatal HF reports reviewed by the ALLHAT Endpoints Subcommittee was limited in number and in adequacy of corroborating documentation, the review nonetheless showed adherence to study criteria for the majority ( $85 \%)$ of the event reports. The sometimes-discrepant results between reviewers pertained more often to incomplete data than to rejection of a HF diagnosis.

Clinical use of more objective measures, including noninvasive and/or invasive tools for the measurement of left ventricular function, offers a means of establishing and quantifying systolic failure in cases clinically suggestive of
HF $[7,10]$. Among the ejection fractions reported in the ALLHAT events that were reviewed, the majority fell at or below $40 \%$, indicating some degree of systolic dysfunction. However, HF is a clinical diagnosis that does not necessarily exclude those with intact left ventricular systolic function [11]. Hypertension is a major risk factor for diastolic HF; as many as $25 \%$ of asymptomatic hypertensives with left ventricular hypertrophy have diastolic dysfunction. Additionally, $90 \%$ of patients with coronary artery disease may have some degree of diastolic dysfunction [12].

The two-fold increased relative risk of HF in the doxazosin group compared to the chlorthalidone group changed little when confined to hospitalized and fatal events. Further, HF patients in both the doxazosin and chlorthalidone treatment groups showed a rather high $20 \%$ case-fatality rate over two years, as expected in HF patients $[11,13,14]$, further supporting the validity of the diagnoses in the two groups. Among participants hospitalized for HF who subsequently died, over half of the deaths in each drug group were attributed to cardiovascular causes.

Treatment group differences in mortality attributed to HF may take time to be recognized. Patients with HF are at risk for other competing causes of death. Accordingly, it may be too early to determine if a higher rate of HF in the doxazosin group translates into a higher overall mortality rate. A $20 \% 2$-year case-fatality rate with a $4 \%$ difference in $\mathrm{HF}$ incidence rate translates into a $0.8 \%$ potential difference in total mortality without any competing causes of 


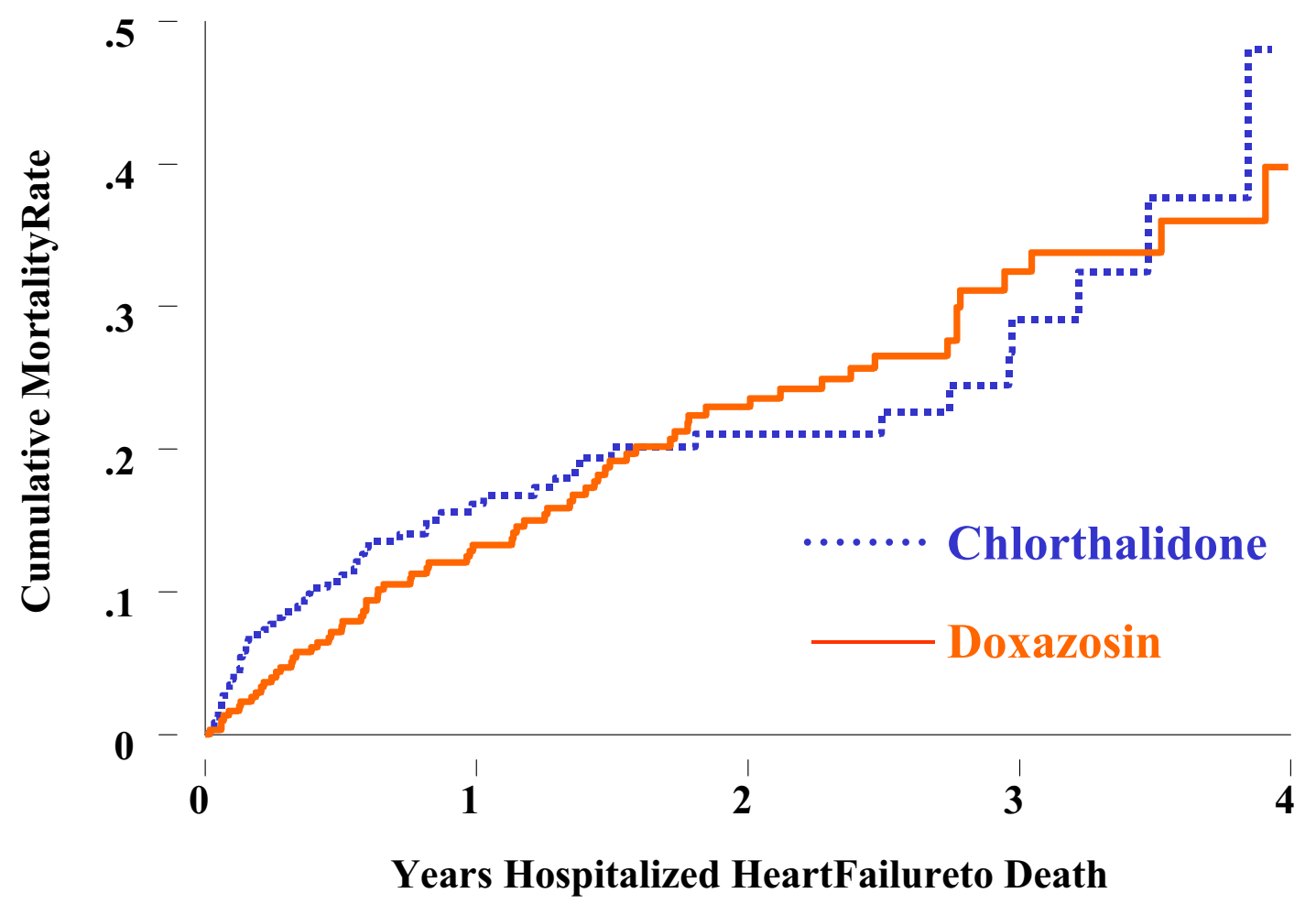

Figure I

Kaplan-Meier estimates for cumulative mortality for hospitalized heart failure cases in the chlorthalidone and doxazosin groups (RR 0.96, 95\% Cl, 0.67-I.38; $P=0.84$ )

death. With competing causes, the difference would be smaller and difficult to detect even in a trial of ALLHAT's size.

The diagnosis of HF is generally made on the basis of signs and symptoms that may overlap with those of other cardiovascular and pulmonary pathologies. Moreover, the clinical picture may be further complicated for patients taking doxazosin, side effects of which (edema, dyspnea, and tachycardia) [15] may be misinterpreted as manifestations of HF.

The capture of events in a "large and simple trial" such as ALLHAT has potential limitations. Built into a structure composed largely of community-based physicians is the assumption that their characterization of clinical events reflects diagnostic standards of the medical community, and, as such, meets study criteria. However, with its large number of endpoints, resources available to ALLHAT pre- cluded more than modest confirmatory event documentation. Resources were allocated for additional documentation for quality control validation only for a sample of the primary endpoint (MIs and fatal CHD) and for strokes. While all reported events are reviewed at the CTC, the sometimes incomplete supporting details in documentation may not allow for validation of all events according to ALLHAT criteria. Some discharge summaries and death certificates may lack sufficient descriptive information needed to confirm the clinical diagnoses. Clinic staff are unable to provide corroborating documentation for $2 \%$ of ALLHAT event reports.

Efforts to authenticate the HF events in the doxazosin and chlorthalidone treatment groups represented a desirable step in the examination of the increased rate of HR in the doxazosin group. All methods employed provided confirmatory evidence that both the HF diagnosis and the difference in HF rates noted between the doxazosin and 
chlorthalidone groups were valid. Results of this exploratory investigation support the event ascertainment methods developed for ALLHAT, specifically for HF events. This validation exercise further illustrates the ability of large, simple trials to answer important public health questions requiring large sample sizes and to grapple with unexpected results in a responsible and meaningful manner.

\section{Competing Interests}

The authors of this paper disclose their affiliations with Aventis (A), Abbott (AB), AstraZeneca (AZ), Bayer (B), Biovail (BV), Bristol-Myers Squibb (BMS), Forest (F), GlaxoSmithKline/SmithKline Beecham (GSK/SKB), King Pharmaceuticals/Monarch (KM), Merck (M), Novartis (N), Nu-Pharm (NP), Pharmacia/Upjohn (PHU), Pfizer, Inc. (P), Sankyo (SY), Searle (SE), Solvay (SV), Takeda (T). These relate to personal or institutional-affiliated receipt of income in the areas of research grants, consultant fees, or other compensation: BRD (AB,BMS,F,M,P,PHU,GSK/ SKB), WCC (AZ,BMS,F,M,P,PHU,SY,SE,SV,T), JTW $(\mathrm{A}, \mathrm{B}, \mathrm{BMS}, \mathrm{F}, \mathrm{KM}, \quad \mathrm{M}, \mathrm{N}, \mathrm{P}), \quad$ FHHL (AZ,B,BMS,M,NP,P,PHU), LJH (PHU); the other authors report no competing interests.

\section{Additional Information}

1) New York Heart Classification functional class III: "Patients with cardiac disease resulting in marked limitation of physical activity. They are comfortable at rest. Less than ordinary physical activity causes fatigue, palpitation, dyspnea, or anginal pain." 1994 Revisions to Classification of Functional Capacity and Objective Assessment of Patients with Diseases of the Heart: AHA Medical/Scientific Statement.

2) The ALLHAT Manual of Operations provides for prescription of open-label medicines of the same class as the blinded medications, when a compelling indication exists, such that the total dose of the added open-label drug should not exceed $1 / 2$ of the maximum dose as recommended in the Sixth Report of the Joint National Committee on Detection, Evaluation and Treatment of High Blood Pressure (JNC VI). If a compelling reason requires a higher dose, it is permitted.

3) The ALLHAT follow-up form allows for reporting of open-label ACE-inhibitors and diuretics; among betablockers, only atenolol is reported. The use of other betablockers cannot be ascertained from the data.

\section{Acknowledgments}

This study is supported by a contract with the National Heart, Blood, and Lung Institute (NHLBI). The ALLHAT investigators acknowledge contributions of study medications supplied by Pfizer, Inc. (amlodipine and doxazosin), AstraZeneca (atenolol and lisinopril), and Bristol-Myers Squibb (pravastatin), and financial support provided by Pfizer to the NHLBI.

\section{References}

I. Davis BR, Cutler JA, Gordon D, for the ALLHAT Research Group, et al: Rationale and design of the Antihypertensive and Lipid Lowering Treatment to Prevent Heart Attack Trial (ALLHAT). Am J Hypertens 1996, 9:342-360

2. The ALLHAT Officers and Coordinators for the ALLHAT Collaborative Research Group: Major cardiovascular events in hypertensive patients randomized to doxazosin vs. chlorthalidone: The Antihypertensive and Lipid-Lowering Treatment to Prevent Heart Attack Trial (ALLHAT). JAMA 2000, 283:19671975

3. Furberg CD, Cutler JA, Davis BR: Status of alpha-blocker therapy in the treatment of hypertension: Findings of ALLHAT. Harrison's Online. The Mc-Graw-Hill Companies 200 l update article to Chapter 246

4. Pressel SL, Davis BR, Wright JT, et al: Operational aspects of terminating the doxazosin arm of the Antihypertensive and Lipid Lowering Treatment to Prevent Heart Attack Trial (ALLHAT). Control Clin Trials 200I, 22:29-4I

5. Kostis JB, Davis BR, Cutler J: Prevention of heart failure by antihypertensive drug treatment in older persons with isolated systolic hypertension. JAMA 1997, 278:212-216

6. Braunwald E, Editor: Heart Disease: A Textbook of Cardiovascular Medicine W.B. Saunders Co 1997, 47I-49I

7. Williams JF, Bristow MR, Fowler MB, et al: Guidelines for the evaluation and management of heart failure: Report of the American College of Cardiology/American Heart Association Task Force on Practice Guidelines (Committee on Evaluation and Management of Heart Failure). Circulation 1995, 92:2764-2784

8. Changes in mortality from heart failure - United States, | 980-|995 CDC MMWR Weekly 1998, 47:633-637

9. Kronstam MA: Progress in heart failure management? Lessons from the real world (editorial). Circulation 2000, 102:1076-1078

10. Shamsham F, Mitchell J: Essentials of the diagnosis of heart failure. Am Family Physician 2000, 6 I: I3 19-1328

II. Vasan RS, Larson MG, Benjamin EJ, et al: Congestive heart failure in subjects with normal versus reduced left ventricular ejection fraction: Prevalence and mortality in a population-based cohort. J Am Coll Cardiol 1999, 33:1948-1955

12. Mandinov L, Eferli FR, Seiler C, et al: Diastolic heart failure (review). Cardiovasc Res 2000, 45:8I3-825

13. Maclntire K, Capewell S., Stewart JWT, et al: Evidence of improving prognosis in heart failure: Trends in case fatality in $\mathbf{6 6 5 4 7}$ patients hospitalized between 1986 and 1995. Circulation 2000, 102: | |26-I|3|

14. Ho KKL, Anderson KM, Kannel WB, et al: Survival after the onset of congestive heart failure in Framingham Heart Study subjects. Circulation 1993, 88:107-115

15. Physician's Desk Reference. 200I, 2475-2476

\footnotetext{
\begin{tabular}{|} 
Publish with BioMed Central and every \\
scientist can read your work free of charge \\
"BioMedcentral will be the most significant development for \\
disseminating the results of biomedical research in our lifetime." \\
Paul Nurse, Director-General, Imperial Cancer Research Fund \\
Publish with BMC and your research papers will be: \\
- available free of charge to the entire biomedical community \\
- peer reviewed and published immediately upon acceptance \\
- cited in PubMed and archived on PubMed Central \\
- yours - you keep the copyright \\
Submit your manuscript here: \\
http://www.biomedcentral.com/manuscript/
\end{tabular}
} 